\title{
Impact of Luteal phase clomiphene citrate on endometrial thickness and clinical pregnancy rates in women with polycystic ovary syndrome
}

\begin{abstract}
Objective: To compare between luteal phase clomiphene citrate and second cycle day clomiphene citrate on the thickness of the endometrium and clinical pregnancy rates in women with polycystic ovary syndrome.

Methods: a prospective observational study conducted on 196 women with Polycystic ovary syndrome divided into two groups: Group 1 (98 patients) received 100mg of clomiphene citrate daily for 5 days starting on second cycle day and Group 2 (98 patients) received $100 \mathrm{mg}$ of clomiphene citrate daily starting in the luteal phase before onset of withdrawal bleeding for 5 days. Treatment and follow up applied for three cycles. Outcome measures were collected and analyzed and women who got pregnant were followed until 12 weeks of pregnancy.
\end{abstract}

Results: no significant differences between the two groups regarding pre-ovulatory endometrial thickness ( $8.3 \mathrm{~mm}$ in group 1vs $8.8 \mathrm{~mm}$ in group 2), number of mature follicles (1.62 in group 1vs 1.57 in group 2) and mid-luteal serum progesterone levels $(14.63 \mathrm{ng} / \mathrm{ml}$ in group $1 \mathrm{vs} 14.9 \mathrm{ng} / \mathrm{ml}$ in group 2). 34 women (35\%) got pregnant in group 1 and 37 women $(38 \%)$ in-group 2 and three patients had miscarriage in each group without any statistical significance.

Conclusion: the present study does not support luteal clomiphene citrate over second cycle day clomiphene citrate to induce ovulation in infertile women with polycystic ovary syndrome.

Keywords: clomiphene citrate, polycystic ovary syndrome, ovulation induction, luteal clomiphene citrate
Volume II Issue 3 - 2020

\author{
Elsayed Elshamy, ${ }^{1,2}$ Ayman Soliman' \\ 'Department of Obstetrics and Gynecology, Faculty of Medicine, \\ Menoufia University, Egypt \\ ${ }^{2}$ Department of Obstetrics and Gynecology, King Abdul-Aziz \\ Airbase hospital, Saudi Arabia
}

\begin{abstract}
Correspondence: Elsayed Elshamy, MD, Department of Obstetrics and Gynecology, King Abdul-Aziz Airbase hospital, Dhuhran, 041/9 Prince Sattamstreet,Al-Khobar, Saudi Arabia, Tel+966534547838, Email s_shamy77@yahoo.com
\end{abstract}

Received: April 14, 2020 | Published: May 19, 2020

\section{Introduction}

Infertility affects $15 \%$ of couples. ${ }^{1}$ Polycystic ovary syndrome (PCO) constitutes a major cause of infertility due to ovarian factor, either anovulation or oligovulation, being the most common endocrine disorder during childbearing period, up to $95 \%$ of ovarian factors of infertility are attributed to PCO., ${ }^{2,3}$

In women with $\mathrm{PCO}$, follicular maturation is arrested resulting in multiple small follicles four to eight $\mathrm{mm}$ in diameter and no dominant follicle leading to poor ovulation, oligo- and/or amenorrhea and infertility. ${ }^{4}$ The first-line treatment for anovulatory women with PCO is to induce ovulation with clomiphene citrate (CC) being the first drug of choice for such disorder. ${ }^{5}$ Fertilized ovum and proper endometrium are required to get conception. With CC, ovulation rates ranges between 60 and $85 \%$ but, unfortunately, conception rates are $20-40 \%{ }^{6}$ This could be explained by the negative impact on the endometrium and cervical mucus with possible effects on oocytes and granulose cells. These effects are prolonged by the relatively long half- life of CC (5days). ${ }^{6,7}$ The endometrial thickness during the peri-ovulatory period is reported to be correlated with successful implantation and pregnancy outcomes. ${ }^{8,9}$ Early initiation of CC to induce ovulation in $\mathrm{PCO}$ women in the luteal phase was first suggested in2009 and reported increased follicular growth and endometrial thickness and pregnancy rates. ${ }^{10}$ And relatively more recent studies supported the promising outcomes of luteal CC in PCO women. ${ }^{6,11}$
The aim of the study was to compare between the impact of luteal phase clomiphene citrate versus second cycle day clomiphene citrate on endometrial thickness and clinical pregnancy rates when used for ovulation induction in women with PCO.

\section{Materials and methods}

This prospective observational study was conducted at Menoufia university Hospital, Menoufia governorate, Egypt between March 2017 and July 2019, on 196 infertile women with PCO, recruited from the Outpatient Infertility Clinic. All infertile women with PCO attending outpatient clinic during the study period were eligible to participate. After counseling and full explanation of the study objectives, informed consent was signed by women who accepted to participate (Figure 1: flow diagram). PCO was diagnosed according to the criteria of Rotterdam consensus. ${ }^{12}$ This study followed the Ethical Committee rules of Obstetrics and Gynecology in Menoufia University Hospital and approved by the study ethical committee of Menoufia faculty of medicine. Inclusion criteria included PCO women aged 18-35years, normal uterus and tubes proved by Hysterosalpingography (HSG), husband with normal semen analysis according to WHO 2010 criteria, ${ }^{13}$ normal baseline prolactin level and normal thyroid profile. Women with growth pelvic lesions were excluded from the study. For all participants, qualitative serum beta HCG was tested to exclude pregnancy just before initiating withdrawal bleeding using dydrogesterone $10 \mathrm{mg}$ orally twice daily(Duphastone $10 \mathrm{mg}$, Abbott 
Egypt, New Cairo, Egypt) for 5 days before stimulation. Participants who fulfilled inclusion criteria were given successive numbers from one to 196 according to their attendance to outpatient clinic then they were divided into two equal groups; group $\mathbf{1}$ (with numbers $1,3,5$... etc) and group 2 ( with numbers $2,4,6 \ldots$ etc). The patients in Group 1 (98patients, 265 cycles) received 100mg of CC (Clomid $50 \mathrm{mg}$ tab, Aventis, Egypt) daily for 5 days starting on day 2 of menses, and those in Group 2 (98 patients, 261 cycles) received $100 \mathrm{mg}$ of CC daily starting on the day just after finishing dydrogesterone and before bleeding for 5 days. A transvaginal ultrasound (TVS) was performed before initiation of treatment to determine the mean follicular number and the baseline endometrial thickness, then on day 10 then serially as required until the size of the dominant follicle reaches $18 \mathrm{~mm}$. At that time, endometrial thickness was measured and Human and chorionic gonadotropin injection 10,000 International Units hCG; (Profasi $H P ®$, Serono S. A., Geneva, Switzerland) was given by intramuscular injection. Patients were advised to have intercourse 24-36 hours after hCG injection serum Progesterone (in nanograms per milliliter) was measured 8 days after hCG injection. Serum beta-hCG was measured
2 weeks after hCG injection in the absence of menstruation for diagnosis of pregnancy. For those with biochemical pregnancy, TVS was done after 2 weeks to diagnose clinical pregnancy then regular follow up until 12 weeks to detect miscarriage. If no dominant follicle detected until day 15 of treatment, serial transvaginal ultrasound until day 20, if still no dominant follicle, the patient was considered as failed induction. The same regimen was repeated for each woman, unless got pregnant, for three cycles. The endometrial thickness was measured in the sagittal view as the maximum thickness between the highly reflective interface of the endometrial-myometrial junction. Regarding endometrial thickness, antral follicle count (AFC), the number of mature follicles and mid- luteal serum progesterone, the mean over the 3 cycles was taken and if pregnancy occurred after one cycle, only single reading was taken and if pregnancy occurred after two cycles, the mean value for the two cycles was calculated. The primary outcome measures were the endometrial thickness and Clinical pregnancy rate. The secondary outcome measure was the number of mature follicles, mid-luteal serum progesterone and the occurrence of miscarriage.

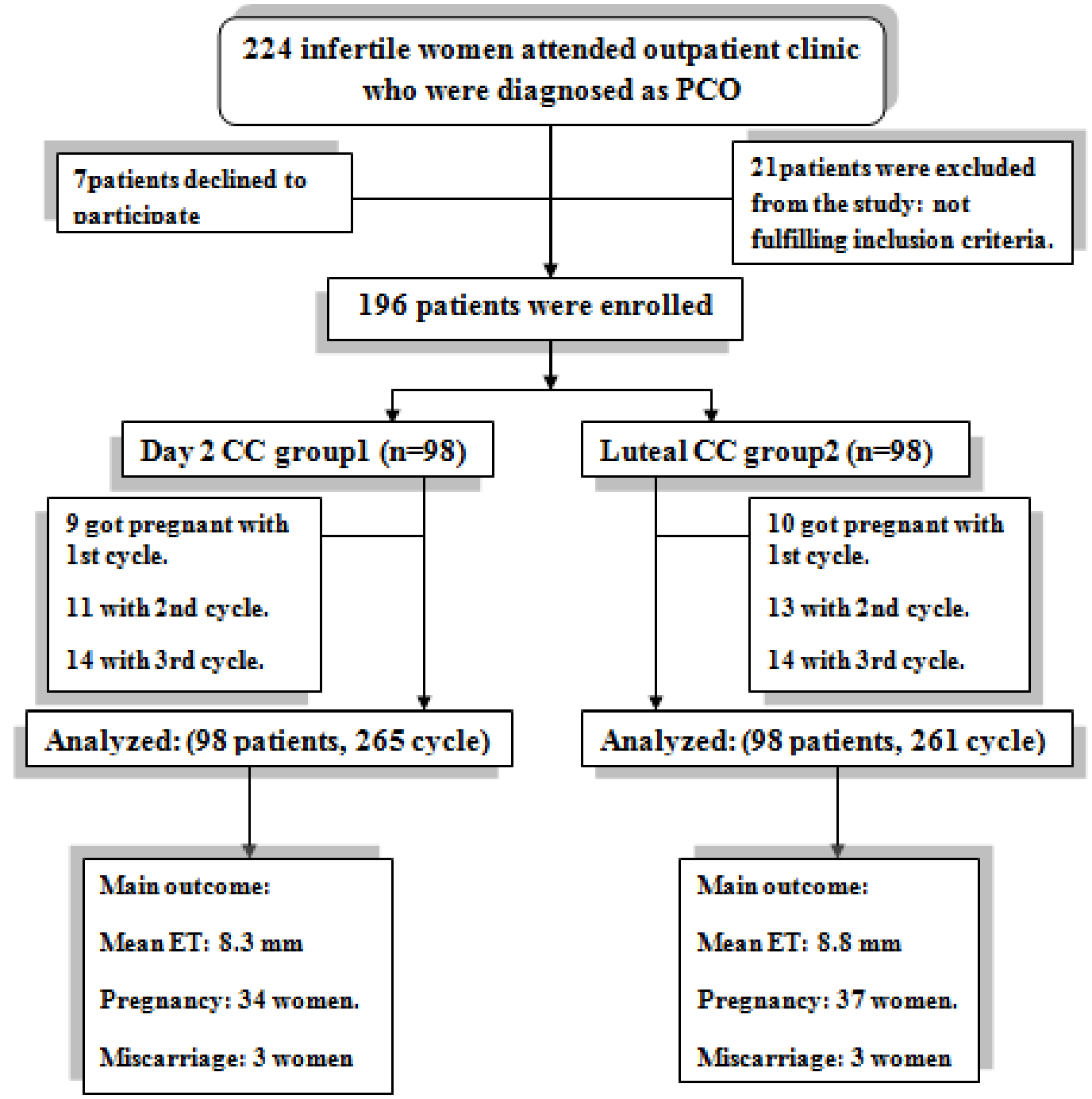

Figure I Flow diagram of patients in the study. 
Statistical analysis was done using SPSS version 20(SPSS Inc., Chikago, IL, USA). Normally distributed numerical data were presented as mean and standard deviation. Qualitative data were presented as the number and percentage. Numerical data were compared with unpaired Student's $t$-test. Chi-Squared $\left(\chi^{2}\right)$ was used for qualitative variables. Odd's Ratio (OR) with $95 \%$ confidence interval (CI) was evaluated for clinical pregnancy rates of both groups. Relative Risk (RR) with 95\% CI was measured for miscarriage rates. $P$ value $<0.05$ is considered significant.

\section{Results}

The two groups were matched for age (mean age 24.8 vs. 25.1 years) and body mass index (BMI) (mean BMI 25.3 vs. $25.7 \mathrm{Kg} / \mathrm{M}^{2}$ ) in group 1 and group 2 respectively.

There were no statistically significant differences between the two groups regarding patient characteristics in terms of age, duration of infertility, basal FSH and LH and antral follicle count (AFC) as revealed in Table 1.

Table I Patient characteristics

\begin{tabular}{lllll}
\hline & Group I (N: 98) & Group 2 (N: 98) & T test & P value \\
\hline Age $(\mathrm{y})$ & $24.8 \pm 2.72$ & $25.1 \pm 2.76$ & 0.77 & 0.4 \\
Duration of infertility $(\mathrm{y})$ & $4.2 \pm 1.38$ & $4.5 \pm 1.42$ & 1.5 & 0.14 \\
$\mathrm{BMI}\left(\mathrm{Kg} / \mathrm{M}^{2}\right)$ & $25.3 \pm 3.8$ & $25.7 \pm 4.44$ & 0.68 & 0.5 \\
$\mathrm{FSH}(\mathrm{mlU} / \mathrm{ml})$ & $6.7 \pm 1.18$ & $6.2 \pm 1.22$ & 1.75 & 0.08 \\
$\mathrm{LH}(\mathrm{mlU} / \mathrm{ml})$ & $7.9 \pm 2.14$ & $8.3 \pm 1.9$ & 1.38 & 0.17 \\
AFC & $14.7 \pm 3.1$ & $14.8 \pm 3.22$ & 0.22 & 0.8
\end{tabular}

$\mathrm{BMI}$, body mass index; FSH, follicle stimulating hormone; $\mathrm{LH}$, luteinizing hormone; $\mathrm{AFC}$, antral follicle count

Table 2 shows no significant differences between the two groups regarding basic endometrial thickness (ET). ET at hCG injection ( $8.3 \mathrm{~mm}$ in group $1 \mathrm{vs} 8.8 \mathrm{~mm}$ in group 2$)$, mean number of mature follicles (1.62 in group 1vs 1.57 in group 2) and mid-luteal serum progesterone levels $(14.63 \mathrm{ng} / \mathrm{ml}$ in group $1 \mathrm{vs} 14.9 \mathrm{ng} / \mathrm{ml}$ in group 2).
Table 3 shows a little, non significant, increase in clinical pregnancy rates in group 2 (34 women (35\%) got pregnant in group 1 vs. 37 women (38\%) in group) 2 and miscarriage rates were comparable in women who got pregnant in both groups (3women $(0.9 \%)$ in group 1 and 3 women $(0.8 \%)$ in group 2$)$.

Table 2 Comparison between the studied groups regarding number of mature follicles, endometrial thickness and mid-luteal serum progesterone

\begin{tabular}{lllll}
\hline & Group I (N: 98) & Group 2 (N: 98) & T test & P value \\
\hline Number of mature follicles & $1.62 \pm 0.87$ & $1.57 \pm 0.91$ & 0.4 & 0.7 \\
Basic ET $(\mathrm{mm})$ & $2.97 \pm 0.54$ & $3.1 \pm 0.5 \mathrm{I}$ & 1.73 & 0.08 \\
ET at hCG injection $(\mathrm{mm})$ & $8.3 \pm 0.43$ & $8.8 \pm 0.48$ & 0.12 & 0.9 \\
Progesterone level $(\mathrm{ng} / \mathrm{mL})$ & $14.63 \pm 10.2$ & $14.9 \pm 11.3$ & 0.18 & 0.9 \\
\hline
\end{tabular}

ET, endometrial thickness

Table 3 Comparison between the studied groups regarding clinical pregnancy rate, and miscarriage rate

\begin{tabular}{llllll}
\hline & Group I (N: 98) & Group 2 (N: 98) & Chi square & P value \\
\hline Clinical pregnancy rate & $34(35 \%)$ & $37(38 \%)$ & 0.08 & 0.77 & OR at $95 \% \mathrm{Cl}:$ \\
& & & & $1.14(0.64-2.05)$ \\
Miscarriage rate & $3(0.9 \%)$ & $3(0.8 \%)$ & 0.49 & 0.48 & $\mathrm{RR}$ at $95 \% \mathrm{Cl}:$ \\
& & & & $0.92(0.2-4.25)$
\end{tabular}

\section{Discussion}

The discrepancy between ovulation and pregnancy rates in PCO women induced by $\mathrm{CC}$ is still a matter of research. Women who take CC have a negative impact on cervical mucus and endometrial thickness. $^{3}$ Trials to overcome these effects were to recommend intrauterine insemination and/or to give a course of estradiol in the late follicular phase, but without significant benefits. ${ }^{6}$ The current study evaluated the impact of luteal CC on endometrial thickness and clinical pregnancy rates in infertile women with PCO compared to traditional day $2 \mathrm{CC}$. There was a small positive effect on endometrial thickness and clinical pregnancy rates with luteal $\mathrm{CC}$ but without statistical significance and the overall outcomes were comparable between the two groups. Based on the negative impact of $\mathrm{CC}$ on cervical mucus and endometrial thick and it is long half-life, some reports detected a higher implantation rates and pregnancy rates when $\mathrm{CC}$ was started on day 1 of the menstrual cycle compared to traditional Day 5 CC therapy. ${ }^{14,15}$

Badawy et al., ${ }^{10}$ presented the first report for comparison between luteal $\mathrm{CC}$ and day $5 \mathrm{CC}$ to induce ovulation in PCO women, where they studied 212 patients (110 with luteal CC and 102 with day 5 CC) 
and they found significant increase in ET at time of hCG injection, contrary to finding of the current study. Regarding pregnancy rates they found relatively more pregnancy rates in luteal $\mathrm{CC}$ group in comparison to the current study which detected little increase in pregnancy rates in luteal CC group although differences in both groups were not statistically significant. This difference can be attributed to comparing luteal CC to cycle day $5 \mathrm{CC}$ in their study versus cycle day 2 in the current study giving shorter interval between the two regimens and relatively longer time from hCG triggering in the current study.

Kosar et al., ${ }^{6}$ conducted a study comparing luteal $\mathrm{CC}$ to cycle day $5 \mathrm{CC}$ and reported, in contrary to the current study, significantly higher number of mature follicles and increased ET at hCG injection in the luteal $\mathrm{CC}$ group. Again, these differences can be attributed to comparing luteal $\mathrm{CC}$ to cycle day $5 \mathrm{CC}$ in their study versus cycle day 2 in the current study. On the other hand, they reported no significant differences in pregnancy rates between the two regimens and no significant differences in miscarriage rates in women who got pregnant between both groups which is coincident with the current study.

A recent systematic review and meta-analysis concluded, according to available evidence, that induction of ovulation using early (luteal $\mathrm{CC}$ ) was associated with more follicular growth and increased ET that might improve pregnancy rate with hopeful reduction in miscarriage rates but still lacking the power to establish a firm conclusion. ${ }^{11}$

Regular follow up for all participants for 3 cycles and for those who got pregnant until 12 weeks and small number of dropout cases constitute the points of strength in this study.

Inability to conduct a randomized trial constitutes unintended limitations of the study.

In Future research, a multicenter, intensive randomized controlled trials should be conducted to define more eligible evidence for luteal phase CC administration to induce ovulation in women with PCO.

\section{Conclusion}

The current study does not support the luteal $\mathrm{CC}$ over cycle day 2 $\mathrm{CC}$ to induce ovulation in infertile women with PCO.

\section{Acknowledgments}

The authors would like to acknowledge the cooperation of the nursing staff in the outpatient clinic of Menoufia university hospital.

\section{Funding}

None.

\section{Conflicts of interest}

The authors declare that no conflicts of interest in relation to this article exist.

\section{References}

1. Propst AM, Bates GW. Evaluation and treatment of anovulatory and unexplained infertility. Obstet Gynecol Clin N Am. 2012;39(4):507-519.

2. Anwar A, Torky H, Dief O, et al. The effect of clomiphene citrate versus Tamoxifen versus letrozoleon endometrial thickness and Blood flow in ovulation induction in women with polycystic ovaries. Acta Medica International. 2016;3(2):88-92.

3. Elshamy E, khalafallah M. Impact of clomiphene citrate, tamoxifen and letrozole to induce ovulation in anovulatory women with polycystic ovary syndrome on endometrial thickness and clinical pregnancy rates, a two center cohort study. Obstet Gynecol Int J. 2018;9(4):260-264.

4. Teede H, Deeks A, Moran L. Polycystic ovary syndrome: a complex condition with psychological, reproductive and metabolic manifestations that impacts on health across the lifespan. BMC Med. 2010;8:41.

5. Fields E, Chard J, James D, et al. Fertility (update): summary of NICE guidance. BMJ. 2013;346:f650.

6. Kosar O, Ozaksit G, Taskin MI. Luteal phase clomiphene citrate for ovulation induction in women with polycystic ovary syndrome. Arch Gynecol Obstet. 2014;290(4):771-775.

7. Dickey RP, Holtkamp DE. Development, pharmacology and clinical experience with clomiphene citrate. Hum Reprod Update. 1996;2(6):483506.

8. Kumbak B, Erden HF, Tosun S, et al. Outcome of assisted reproduction treatment in patients with endometrial thickness less than $7 \mathrm{~mm}$. Reprod Biomed Online. 2009;18(1):79-84.

9. Kasius A, Smit JG, Torrance HL, et al. Endometrial thickness and pregnancy rates after IVF: a systematic review and meta-analysis. Hum Reprod Update. 2014;20(4):530-541.

10. Badawy A, Inany $\mathrm{H}$, Mosbah $\mathrm{A}$, et al. Luteal phase clomiphene citrate for ovulation induction in women with polycystic ovary syndrome: a novel protocol. Fertil Steril. 2009;91(3):838-841.

11. Ding N, Chang J, Jian Q, et al. Luteal phase clomiphene citrate for ovulation induction in women with polycystic ovary syndrome: a systematic review and meta-analysis. Gynecol Endocrinol. 2016;32(11):866-871.

12. Rotterdam ESHRE/ASRM-Sponsored PCOS Consensus Workshop Group. Revised 2003 consensus on diagnostic criteria and longterm health risks related to polycystic ovary syndrome. Fertil Steril. 2004;81(1):19-25.

13. The Practice Committee of the American Society for Reproductive Medicine. Diagnostic evaluation of the infertile male: A committee opinion. Fertil Steril. 2012;98(2):294-301.

14. Kelekçi S, Saygili-Yilmaz E, Inan I, et al. A trial of a new regimen with clomiphene citrate administration to reduce the antiestrogenic effects on reproductive end organs. Eur J Obstet Gynecol Reprod Biol. 2004;10(116):54-57.

15. Dehbashi S, Vafaei H, Parsanezhad MD, et al. Time of initiation of clomiphene citrate and pregnancy rate in polycystic ovarian syndrome. Int J Gynaecol Obstet. 2006;93(1):44-48. 